\title{
Insuficiencia velofaríngea en cirugía adenoamigdalina
}

\author{
Velopharyngeal insufficiency in adenotonsillar surgery
}

\author{
Changhua Lu¹, Claudia Arriagada G'1 Edda Pruzzo $\mathrm{Ch}^{1}$, Cecilia Gallo J², Verónica Bley P².
}

\begin{abstract}
RESUMEN
Introducción: Insuficiencia velofaríngea (IVF) es una posible complicación asociada a cirugía adenoamigdalina, cuya incidencia real es difícil de establecer, según la literatura, su frecuencia estimada es de 1 en 1.500-10.000 adenoidectomías. Sin embargo, no hay registro en la literatura de IVF transitoria posoperatoria. Nuestra hipótesis del trabajo: la IVF posoperatoria, tanto transitoria como definitiva, está subdiagnosticada 0 subregistrada.

Objetivo : Evaluar la frecuencia y estudiar los factores predisponentes de IVF transitoria y definitiva posoperatoria en una población del Área Occidente de la Región Metropolitana de Chile, 2004-2007.

Material y método: Estudio descriptivo y retrospectivo, Revisión de fichas clínicas y registros de la Unidad de Fonoaudiología de pacientes <15 años, operados de patología adenoamigdalina en el Servicio de Otorrinolaringología del Hospital San Juan de Dios, 2004-2007.

Resultados: Se registraron 18 casos de IVF transitoria posoperatoria que corresponde a $1,2 \%(n=1.458)$. La frecuencia de IVF definitiva posoperatoria fue cero.

Conclusión: No se logró analizar los posibles factores predisponentes de IVF posoperatoria. El porcentaje de IVF transitoria encontrado constituye una estadística de referencia para la elaboración del consentimiento informado. Es posible que la frecuencia encontrada pueda ser menor a la real debido al inadecuado registro y control posoperatorio. Creemos que es necesario un protocolo de estudio y de seguimiento de IVF posoperatoria en todos los pacientes intervenidos de cirugía adenoamigdalina.

Palabras claves: Insuficiencia velofaríngea posoperatoria, IVF transitoria, IVF definitiva, hipernasalidad.
\end{abstract}

\begin{abstract}
Introduction: Velopharyngeal insufficiency (VPI) is a possible complication associated with adenotonsillar surgery, whose real incidence is difficult to establish, according to the literature, the estimated frequency is 1 in 1500-10000 adenoidectomies. However, there is no record in the literature of transient postoperative VPI. Hypothesis: The postoperative $\mathrm{VPI}$, both transient and permanent, is underdiagnosed and underreported.
\end{abstract}

Médico. Servicio de Otorrinolaringología Hospital San Juan de Dios.

Fonoaudiólogo. Servicio de Otorrinolaringología Hospital San Juan de Dios. 
Aim: To evaluate the frequency and predisposing factors study transient and permanent postoperative VPI in a population of the West Area of the Metropolitan Region of Chile, 2004-2007.

Material and method: A retrospective study, clinical records and records from the Audiology Unit of patients <15 years, operated as adenotonsillar pathology in the Department of Otorhinolaryngology, Hospital San Juan de Dios, from 2004 to 2007.

Results: There were 18 cases of transient postoperative VPI which corresponds to $1.2 \%(n=1458)$. The frequency of permanent postoperative VPI was zero.

Conclusion: It was not possible to analyze the possible predisposing factors for postoperative VPI. The percentage of transient found VPI is a statistical reference for the development of informed consent. It is possible that the frequency found to be lower than actual due to inadequate recording and postoperative control. We believe that we need a study protocol and postoperative monitoring in all VPI patients undergoing adenotonsillar surgery.

Key words: Postoperative velopharyngeal insufficiency, transient VPI, permanent VPI, hypernasality.

\section{INTRODUCCIÓN}

Insuficiencia velofaríngea (IVF) es una condición clínica caracterizada por la incapacidad (transitoria o definitiva) del cierre hermético del velo palatino contra la pared faríngea, que causa habla hipernasal, emisión y turbulencia nasal y regurgitación nasal de líquidos ${ }^{1}$. Dicha IVF es una posible complicación asociada a la cirugía adenoamigdalina, cuya incidencia real es difícil de establecer, según la literatura extranjera. Su frecuencia estimada es de 1 en 1.500-10.000 adenoidectomías ${ }^{1-6}$. Sin embargo, no hay registro en la literatura de IVF transitoria posoperatoria.

El velo palatino y la pared faríngea posterior constituyen un esfínter muscular entre las cavidades oral y nasal, su función primordial es impedir el paso de alimentos y líquidos hacia la cavidad nasal durante la deglución y coordinar el cierre del esfínter según el tipo de fonación, haciendo el habla inteligible. Para este fin, el velo palatino se tensa y se eleva hacia la pared faríngea posterior, y ésta, a su vez, junto con las paredes laterales, se mueve hacia el velo para cerrar el espacio oronasofaríngeo. Según visión endoscópica, hay 4 patrones de cierre del velofaríngeo (Figura 1) ${ }^{7}$.

En la emisión de sonidos nasales $(m, n)$, el esfínter permanece abierto. Durante la emisión de vocales $(a, e, i, 0, u)$, como también la de consonantes oclusivas $(p, t, k)$ y fricativas $(f, s)$, el esfínter se cierra parcialmente o totalmente, permitiendo comprimir el aire bajo el punto de cierre y liberándolo con fuerza.

El diagnóstico de IVF se realiza principalmente mediante la sintomatología, los signos clínicos y la nasofaringolaringoscopía. La videofluoroscopía serviría de estudio complementario, especialmente en niños más pequeños.

En el Hospital San Juan de Dios, la evaluación fonoaudiológica de la función velar se realiza según varios parámetros como se ilustra en la Figura 2.

\section{Hipótesis del trabajo}

La insuficiencia velofaríngea posterior a cirugía adenoamigdalina, tanto transitoria como definitiva, está subdiagnosticada 0 subregistrada.

\section{OBJETIVO}

General: Evaluar la frecuencia y estudiar los factores predisponentes de insuficiencia velofaríngea transitoria y definitiva posterior a cirugía adenoamigdalina en una población del Área Occidente de la Región Metropolitana de Chile, 20042007.

Específicos:

- Determinar la frecuencia de IVF transitoria posoperatoria en una población del Área Occidente de la Región Metropolitana. 


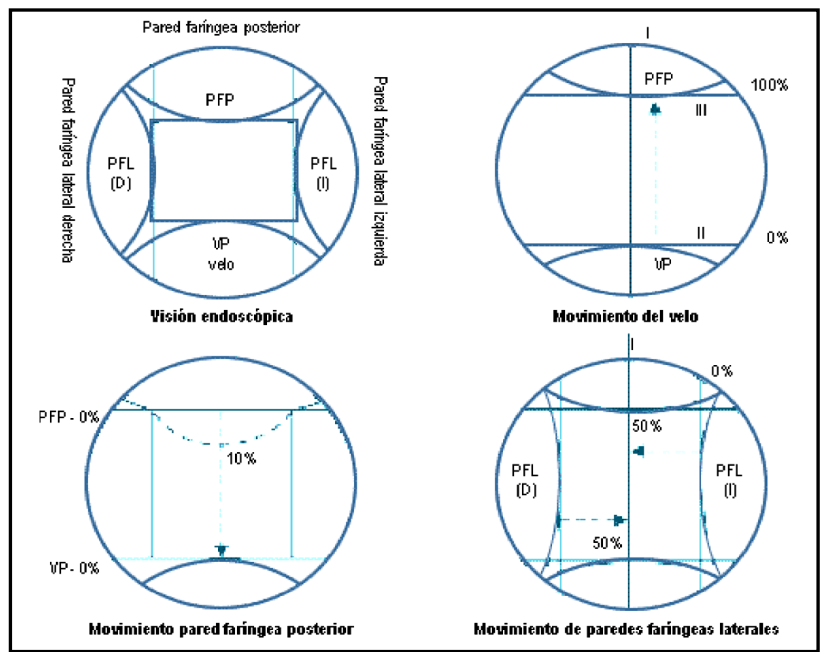

Figura 1. Tipos de patrones de cierre del velo palatino.

Paladar duro:

Velo del paladar:

Úvula:

Voz:

Espejo de Glatzel (+/-): normal - alto - ojival

normal - insuficiente

normal - corta - bífida - ausente

hipernasal - hiponasal

fonema oral con

fonemas oclusivos con

fonemas velares con /a/

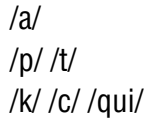

/k/ /c/ /quil/

$\begin{array}{lll}\text { ejemplos: } & \text { papá } & \text { kilo } \\ & \text { pato } & \text { gato } \\ & \text { coca } & \text { plato }\end{array}$

Figura 2. Evaluación fonoaudiológica de la función velar

- Determinar la frecuencia de IVF definitiva posoperatoria en la misma población.

- Evaluar la influencia de los posibles factores predisponentes de IVF

- tamaño amigdalino .

- tamaño adenoídeo .

- grado de adherencia/empotramiento amigdalino.

- técnica quirúrgica (tonsilótomo Daniels, disección fría, electrocauterio).

- cirujano (médico otorrino, residente).

\section{MATERIAL Y MÉTODO}

Se realizó un estudio descriptivo y retrospectivo, estableciendo criterios de exclusión tales como: antecedentes de IVF, úvula bífida, paladar hendido, labio leporino, fisura palatina submucosa, síndrome de Down, anomalías cromosómicas en general, anomalías craneofaciales, trastornos neuro-musculares y patologías psiquiátricas, según se ilustra en la Figura 3 , y definiciones de IVF, tanto transitoria como definitiva 
- Antecedentes de IVF

- Úvula bífida

- Paladar hendido

- Labio leporino

- Fisura submucosa palatina

- Síndrome de Down

- Anomalías cromosómicas en general

- Anomalías craneofaciales

- Trastornos neuromusculares

- Patología psiquiátrica

Figura 3. Criterios de exclusión

(Figura 4). Revisión de fichas clínicas y registros de la Unidad de Fonoaudiología. Los criterios de inclusión consisten en: pacientes con al menos una visita preoperatoria y una posoperatoria, $<15$ años, operados de patología adenoamigdalina en el Servicio de Otorrinolaringología del Hospital San Juan de Dios de Santiago de Chile, 2004-2007. Que para su análisis se dividieron en 3 grupos: 0-5 años, 6-10 años y 11-15 años. Las indicaciones principales son: 1) Hiperplasia adenoamigdalina sintomática, 2) Hiperplasia adenoídea obstructiva, 3) Amigdalitis a repetición, 4) Dos o más abscesos periamigdalinos. Se diseñó una ficha en el Programa Epi info 6.0 para la recopilación de datos, estos últimos se analizaron con estudio de frecuencias, porcentajes y proporciones.

\section{RESULTADOS}

En el período de estudio, de un total de 2.203 pacientes intervenidos de cirugía adenoamigdalina, $1.458(66,2 \%)$ que cumplieron con los criterios de inclusión, corresponden a pacientes $<15$ años que cuentan con evaluación fonoaudiológica antes y después de la cirugía, cuyo rango de edad es entre 3 y 15 años.

Se registraron 18 casos de IVF transitoria posoperatoria que corresponde a $1,2 \%(n=1.458)$. No hubo caso de IVF definitiva posoperatoria.

Al analizar este grupo de 18 pacientes que presentaron IVF transitoria, el $61,1 \%$ corresponde al sexo masculino y el $38,9 \%$ al sexo femenino; la distribución de estos pacientes según grupo etario se muestra en la Figura 5. El grupo de 6-10 años

\section{Definición de IVF transitoria:}

Presencia de hipernasalidad y/o regurgitación nasal de líquidos posterior a cirugía adenoamigdalina de $<3$ meses de duración con 0 sin tratamiento fonoaudiológico.

\section{Definición de IVF definitiva:}

Cuadro clínico de IVF posterior a cirugía adenoamigdalina de $>3$ meses de duración, a pesar del tratamiento fonoaudiológico.

Figura 4.

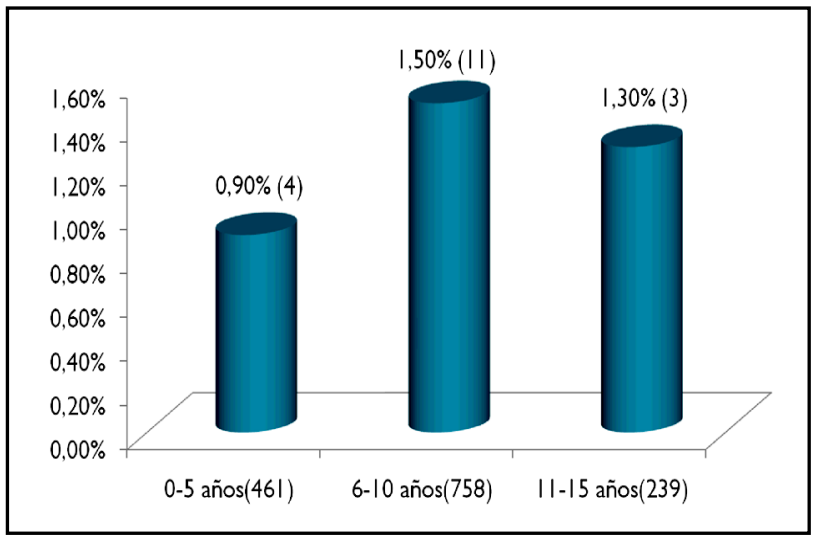

Figura 5. Distribución de pacientes con IVF según grupo etario $(n=18)$. 
presentó la mayoría de casos (11/18), correspondiente a $1,5 \%$ del total. La Figura 6 ilustra la distribución de estos 18 casos según tipos de cirugía. Destaca el hecho que la mayor cantidad de casos se asocian a adenoidectomía, con una incidencia de IVF de $1,4 \%$ en el grupo de las adenoidectomías. La amigdalectomía sola presentó menos porcentaje de casos. En relación a los síntomas, la hipernasalidad estuvo presente en el $61,1 \%$, regurgitación en el $27,8 \%$ y ambos síntomas en el $11,1 \%$; el $72,2 \%$ de estos 18 pacientes recibió tratamiento fonoaudiológico; el restante se recuperó antes del mes después de la cirugía. Cabe señalar que en nuestro hospital, por consenso, se realiza fonoterapia si la IVF persiste por más de un mes posterior a la cirugía (Figura 7). Doce de 18 pacientes (67\%;) fueron intervenidos por médicos otorrinolaringólogos y 6 de 18 por residentes. No fue posible analizar los factores predisponentes de
IVF posoperatoria, ya que estos factores no todos fueron registrados en le ficha clínica.

\section{DISCUSIÓN}

En el período analizado, no encontramos pacientes con IVF definitiva posoperatoria, lo cual concuerda con la frecuencia baja publicada. En nuestra casuística, la IVF transitoria $<3$ meses fue de $1,2 \%$ $(n=1.458)$. Sin embargo, no existen cifras publicadas que permitan su comparación. No hay consenso respecto al momento del inicio del tratamiento fonoaudiológico, algunos autores recomiendan 2 a 3 meses posterior a la cirugía ${ }^{2,8,9}$.

Cabe señalar que este estudio presenta ciertas limitaciones tales como: fichas clínicas con datos e informaciones incompletas; utilización de diversas clasificaciones para algunos parámetros, así como

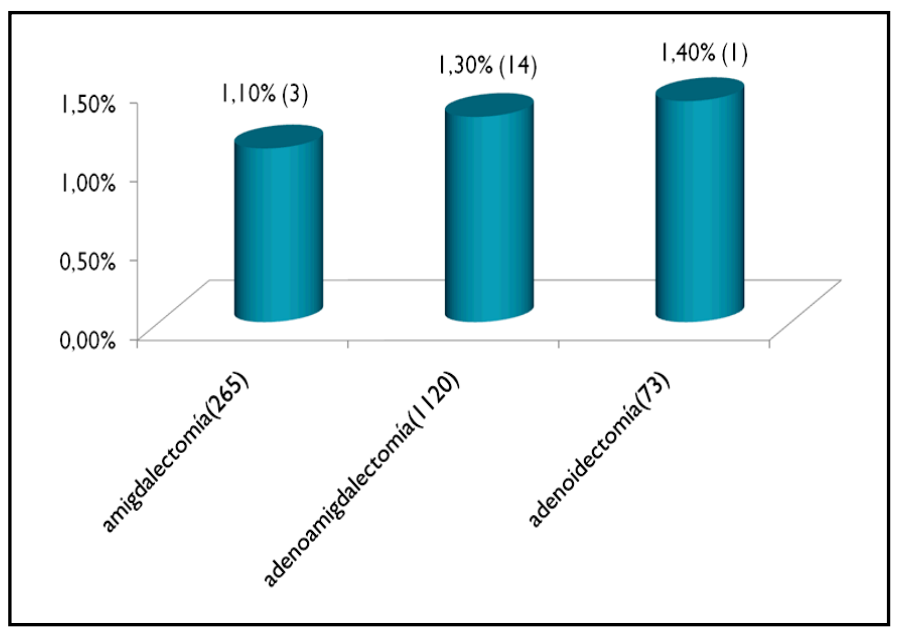

Figura 6. Distribución de pacientes con IVF según tipo de cirugía ( $n=18)$.

- $\quad$ En el HSJD, por consenso, se realiza el tratamiento si la IVF persiste más de un mes posterior a la cirugía

- $\quad$ Ejercicios velares:

- $\quad$ Masaje digital velo palatino

- "Gárgaras" con líquido

- Estimulación del reflejo nauseoso

- $\quad$ Ejecución de fonemas velares y oclusivos

- Técnica respiratoria y de soplo

- $\quad$ Técnica fono-respiratoria

Figura 7. Tratamiento fonoaudiológico de los pacientes con IVF posterior a cirugía adenoamigdalina 
para los factores predisponentes que quisimos estudiar; la evaluación fonoaudiológica se realizó solamente en un grupo de pacientes (1.458 de 2.203); nasofaringolaringoscopía preoperatoria y posoperatoria no fue aplicada a todos los pacientes.

\section{CONCLUSIÓN}

La frecuencia de IVF definitiva posoperatoria en el período analizad o fue cero. La IVF transitoria $<3$ meses fue de $1,2 \%$ ( $n=1.458)$. No se logró analizar los posibles factores predisponentes de IVF posoperatoria. El porcentaje de IVF transitoria encontrado constituye una estadística de referencia para la elaboración del consentimiento informado. Es posible que la frecuencia encontrada pueda ser menor a la real debido al inadecuado registro y control posoperatorio. Creemos que es necesario un protocolo de estudio y de seguimiento de IVF posoperatoria en todos los pacientes intervenidos de cirugía adenoamigdalina.

A nuestra opinión, en base a los resultados de este trabajo, se plantea las siguientes tareas a futuro: 1) obtener clasificaciones consensuadas para los parámetros como tamaño adenoídeo y amigdalino; 2) elaborar registro operatorio estandarizado; 3) crear protocolo de pesquisa postoperatorio.

\section{BIBLIOGRAFÍA}

1. Saunders nC, Hartley BeJ, Sell D, Sommerlad B. Velopharyngeal insufficiency following adenoidectomy. Clin Otolaryngol 2004; 29: 686-8.

2. David A. Randall and Michael E. Hoffer. Complications of tonsillectomy and adenoidectomy. Otolaryngol Head Neck Surg 1998; 118: 61-8.

3. GIBB AG. Hypernasality (Rhinolalia aperta) Following tonsil and adenoid removal. J Laryngol Otol 1958; 72: 433-51.

4. Donnelly M. Hypernasality following adenoid removal. Ir J Med Sci 1994; 163: 225-7.

5. Gelder L. Open nasal speech following adenoidectomy and tonsillectomy. J Commun Disord 1974; 7: 263-7.

6. Witzel M, Rich R, Margar-Bacal F, Cox C. Velopharyngeal insufficiency after adenoidectomy: an 8-year review. Int $J$ pediatr Otorhinolaryngol 1968; 11: 15-20.

7. Rolando Prada M et al. Detección y manejo temprano de la insuficiencia Velofaríngea: una propuesta de manejo integral; Revista Colombiana de Cirugía Plástica y Reconstructiva; Marzo de 2003; vol $9 \mathrm{~N}^{0} 1$.

8. Har-El G, Nash M. Tonsillectomy and adenoidectomy, In: Krespi Y, Ossoff R, editors. Complications in head and neck surgery. Philadelphia: Saunders, 1991; 7598.

9. April M, Naclerio R. Complications of tonsillectomy and adenoidectomy. In: Eisele $\mathrm{D}$, editors. Complications in otolaryngology head and neck surgery. St. Louis: Mosby; 1993; 262-6. 